\title{
MMSE-Based Non-Regenerative Multicarrier MIMO Wireless Relay Communications with Direct Source-Destination Link
}

\author{
Yue Rong \\ Department of Electrical and Computer Engineering, Curtin University of Technology, Bentley, WA 6102, Australia \\ E-mail: y.rong@curtin.edu.au
}

\begin{abstract}
In this paper we propose non-regenerative multicarrier multiple-input multiple-output (MIMO) relay techniques that minimize the mean-squared error (MSE) of the signal waveform estimation. In particular, we consider the practical scenario where the direct source-destination link can not be neglected and develop an alternating technique to minimize the signal MSE. In order to reduce the computational complexity of the alternating algorithm, a suboptimal non-alternating precoding approach is proposed. Numerical examples illustrate a significant performance improvement of the proposed algorithms compared with the existing techniques.
\end{abstract}

\section{INTRODUCTION}

Research on cooperative communications employing relay nodes dates back to 1970s [1]. Recently, cooperative communications have been the subject of renewed research [2]. Both non-regenerative and regenerative cooperative strategies have been developed [2], [3]. In regenerative strategies, the relay node first decodes the received signal, then re-encodes the signal and forward it to the destination node. While in non-regenerative approaches, the relay node only amplifies the received signal and retransmits it. Thus, the non-regenerative approach is also well known as the amplify-and-forward (AF) approach.

In non-regenerative approaches, the relay node does not need to perform decoding and encoding. Therefore, the complexity of the non-regenerative approach is much lower than that of the regenerative approach. This advantage is particularly important when all nodes are equipped with multiple antennas, since decoding multiple data streams involves much more computational efforts than decoding single data stream.

Non-regenerative approaches have been proposed to maximize the mutual information of singlecarrier multiple-input multiple-output (MIMO) relay systems [4], [5] and multicarrier MIMO relay systems [6]. However, in practical communication systems, an important objective is to minimize the transmission error which is closely related to the mean-squared error (MSE) of the signal waveform estimation.

In this paper, we propose non-regenerative multicarrier MIMO relay algorithms to minimize the MSE of the signal waveform estimation. Different to [7], we investigate the practical scenario where the direct source-destination link is present. We show that in this case, the precoding matrices that jointly diagonalize the source-relay-destination channel [7] is no longer optimal. An alternating algorithm is developed to optimize the source and relay precoding matrices. Considering that the computational complexity of the alternating algorithm may be high for practical communication systems, we propose a suboptimal non-alternating approach. In this approach, we first adopt the optimal source precoding matrix that is designed in the absence of the direct source-destination link. Then we efficiently exploit the direct link through optimizing the relay matrix. Compared with the alternating algorithm, the suboptimal approach trades a performance degradation for a tremendously reduced computational complexity.

The rest of this paper is organized as follows. In Section II we introduce necessary background for a multicarrier MIMO relay communication system. The proposed non-regenerative relay approaches are developed in Section III. In Section IV, we show some numerical examples. Conclusions are drawn in Section V.

\section{BACKGROUND}

We consider a three-node multicarrier MIMO communication system where the source node transmits information to the destination node with the aid of one relay node. The source, relay, and destination nodes are equipped with $N_{s}, N_{r}$, and $N_{d}$ antennas, respectively. Due to its merit of simplicity, the nonregenerative strategy is applied at the relay node to process and forward the received signal.

The communication process between the source and destination nodes is completed in two time slots. At the first time slot, the information bits at the source node are encoded by error correction channel codes and modulated. The modulated signal sequence is divided into $N_{c}$ blocks. We denote $N_{b}^{n}$, $n=1, \cdots, N_{c}$ as the number of symbols in the $n$th subblock. Then the source node linearly precodes the $N_{b}^{n} \times 1$ vector of source signal $\mathbf{s}^{n}(t)$ to have

$$
\mathbf{x}^{n}(t)=\mathbf{B}^{n} \mathbf{s}^{n}(t), \quad n=1, \cdots, N_{c}
$$

where $\mathbf{B}^{n}$ is an $N_{s} \times N_{b}^{n},\left(N_{s} \geq N_{b}^{n}\right)$ precoding matrix for the $n$th sub-block of the source signal sequence. The precoded vector of signal $\mathbf{x}^{n}(t)$ is broadcasted to the relay and destination nodes via the $n$th subcarrier. The received signal at the $n$th subcarrier of the relay and destination nodes can be written respectively as

$$
\begin{aligned}
\mathbf{y}_{r}^{n}(t) & =\mathbf{H}_{s}^{n} \mathbf{x}^{n}(t)+\mathbf{v}_{s}^{n}(t), & & n=1, \cdots, N_{c} \\
\mathbf{y}_{d}^{n}(t) & =\mathbf{H}_{d}^{n} \mathbf{x}^{n}(t)+\mathbf{v}_{d}^{n}(t), & & n=1, \cdots, N_{c}
\end{aligned}
$$


where $\mathbf{H}_{s}^{n}$ is an $N_{r} \times N_{s}$ MIMO channel matrix between the source and relay nodes, $\mathbf{H}_{d}^{n}$ is an $N_{d} \times N_{s}$ MIMO channel matrix between the source and destination nodes, $\mathbf{y}_{r}^{n}(t)$ and $\mathbf{v}_{s}^{n}(t)$ are the received signal and the additive Gaussian noise vectors at the relay node, respectively, $\mathbf{y}_{d}^{n}(t)$ and $\mathbf{v}_{d}^{n}(t)$ are the received signal and the additive Gaussian noise vectors at the destination node, respectively. Hereafter, the superscript $n$ denotes the corresponding variables for the $n$th subcarrier.

At the second time slot, the source node is silent. While the relay node multiplies (linearly precodes) the received signal vector at the $n$th subcarrier with an $N_{r} \times N_{r}$ matrix $\mathbf{F}^{n}$ and transmits the precoded signal vector $\mathbf{x}_{r}^{n}(t+1)=\mathbf{F}^{n} \mathbf{y}_{r}^{n}(t)$ to the destination node. The received signal vector at the $n$th subcarrier of the destination node can be written as

$$
\begin{aligned}
\mathbf{y}_{d}^{n}(t+1) & =\mathbf{H}_{r}^{n} \mathbf{x}_{r}^{n}(t+1)+\mathbf{v}_{d}^{n}(t+1) \\
& =\mathbf{H}_{r}^{n} \mathbf{F}^{n} \mathbf{H}_{s}^{n} \mathbf{x}^{n}(t)+\mathbf{H}_{r}^{n} \mathbf{F}^{n} \mathbf{v}_{s}^{n}(t)+\mathbf{v}_{d}^{n}(t+1)
\end{aligned}
$$

where $\mathbf{H}_{r}^{n}$ is an $N_{d} \times N_{r}$ MIMO channel matrix between the relay and destination nodes, $\mathbf{y}_{d}^{n}(t+1)$ and $\mathbf{v}_{d}^{n}(t+1)$ are the received signal and the additive Gaussian noise vectors at the destination node, respectively. We assume that $\mathbf{H}_{s}^{n}, \mathbf{H}_{r}^{n}$, and $\mathbf{H}_{d}^{n}, n=1, \cdots, N_{c}$ are all quasi-static and known by all nodes.

Combining (1)-(4), the received signal vector at the $n$th subcarrier of the destination node over two time slots is

$$
\begin{aligned}
\mathbf{y}^{n}(t) & \triangleq\left[\begin{array}{c}
\mathbf{y}_{d}^{n}(t+1) \\
\mathbf{y}_{d}^{n}(t)
\end{array}\right] \\
& =\left[\begin{array}{c}
\mathbf{H}_{r}^{n} \mathbf{F}^{n} \mathbf{H}_{s}^{n} \\
\mathbf{H}_{d}^{n}
\end{array}\right] \mathbf{B}^{n} \mathbf{s}^{n}(t)+\left[\begin{array}{c}
\mathbf{H}_{r}^{n} \mathbf{F}^{n} \mathbf{v}_{s}^{n}(t)+\mathbf{v}_{d}^{n}(t+1) \\
\mathbf{v}_{d}^{n}(t)
\end{array}\right] .
\end{aligned}
$$

We assume that the additive noise is zero mean, and at each subcarrier of the destination node the noise is temporally white, i.e., $\mathrm{E}\left[\mathbf{v}_{d}^{n}(t)\left(\mathbf{v}_{d}^{n}(t+1)\right)^{H}\right]=\mathbf{0}_{N_{d} \times N_{d}}, n=1, \cdots, N_{c}$, where $\mathrm{E}[\cdot]$ stands for the statistical expectation, and $(\cdot)^{H}$ denotes the matrix Hermitian transpose. Note that if the noise is spatially correlated such that $\mathbf{C}_{v_{s}}^{n} \triangleq \mathrm{E}\left[\mathbf{v}_{s}^{n}(t)\left(\mathbf{v}_{s}^{n}(t)\right)^{H}\right] \neq \mathbf{I}_{N_{r}}$ and $\mathbf{C}_{v_{d}}^{n} \triangleq \mathrm{E}\left[\mathbf{v}_{d}^{n}(t)\left(\mathbf{v}_{d}^{n}(t)\right)^{H}\right] \neq \mathbf{I}_{N_{d}}$, where $\mathbf{I}_{n}$ denotes an $n \times n$ identity matrix, pre-whitening of the received signals can be performed at the relay and destination nodes such that

$$
\tilde{\mathbf{y}}^{n}(t)=\left[\begin{array}{c}
\tilde{\mathbf{H}}_{r}^{n} \tilde{\mathbf{F}}^{n} \tilde{\mathbf{H}}_{s}^{n} \\
\tilde{\mathbf{H}}_{d}^{n}
\end{array}\right] \mathbf{B}^{n} \mathbf{s}^{n}(t)+\left[\begin{array}{c}
\tilde{\mathbf{H}}_{r}^{n} \tilde{\mathbf{F}}^{n} \tilde{\mathbf{v}}_{s}^{n}(t)+\tilde{\mathbf{v}}_{d}^{n}(t+1) \\
\tilde{\mathbf{v}}_{d}^{n}(t)
\end{array}\right]
$$

where

$$
\begin{aligned}
\tilde{\mathbf{H}}_{s}^{n}=\left(\mathbf{C}_{v_{s}}^{n}\right)^{-\frac{1}{2}} \mathbf{H}_{s}^{n}, & \tilde{\mathbf{y}}^{n}(t)=\left(\mathbf{C}_{v_{d}}^{n}\right)^{-\frac{1}{2}} \mathbf{y}^{n}(t), \\
\tilde{\mathbf{H}}_{r}^{n}=\left(\mathbf{C}_{v_{d}}^{n}\right)^{-\frac{1}{2}} \mathbf{H}_{r}^{n}, & \tilde{\mathbf{v}}_{d}^{n}(t+1)=\left(\mathbf{C}_{v_{d}}^{n}\right)^{-\frac{1}{2}} \mathbf{v}_{d}^{n}(t+1), \\
\tilde{\mathbf{H}}_{d}^{n}=\left(\mathbf{C}_{v_{d}}^{n}\right)^{-\frac{1}{2}} \mathbf{H}_{d}^{n}, & \tilde{\mathbf{v}}_{s}^{n}(t)=\left(\mathbf{C}_{v_{s}}^{n}\right)^{-\frac{1}{2}} \mathbf{v}_{s}^{n}(t), \\
\tilde{\mathbf{F}}^{n}=\mathbf{F}^{n}\left(\mathbf{C}_{v_{s}}^{n}\right)^{\frac{1}{2}}, & \tilde{\mathbf{v}}_{d}^{n}(t)=\left(\mathbf{C}_{v_{d}}^{n}\right)^{-\frac{1}{2}} \mathbf{v}_{d}^{n}(t) .
\end{aligned}
$$

From (6) we see that all noises are independent and identically distributed (i.i.d.). Thus, in the following, without loss of generality, we assume i.i.d. Gaussian noise with zero mean and unit variance, and use (5) as the system input-output model.

We assume that a linear receiver is used at the destination node to retrieve the transmitted signals at all subcarriers. The estimated signal waveform is given by

$$
\hat{\mathbf{s}}^{n}(t)=\left(\mathbf{W}^{n}\right)^{H} \mathbf{y}^{n}(t), \quad n=1, \cdots, N_{c}
$$

where $\mathbf{W}^{n}$ is an $2 N_{d} \times N_{b}^{n}$ weight matrix at the $n$th subcarrier.

\section{Minimal MSE (MMSE) Relay Design}

Using the linear receiver (7), the MSE of the signal waveform estimation is

$$
\begin{aligned}
& \sum_{n=1}^{N_{c}} \mathrm{E}\left[\operatorname{tr}\left(\left(\hat{\mathbf{s}}^{n}(t)-\mathbf{s}^{n}(t)\right)\left(\hat{\mathbf{s}}^{n}(t)-\mathbf{s}^{n}(t)\right)^{H}\right)\right] \\
& =\sum_{n=1}^{N_{c}} \operatorname{tr}\left(\left(\left(\mathbf{W}^{n}\right)^{H} \overline{\mathbf{H}}^{n} \mathbf{B}^{n}-\mathbf{I}_{N_{b}^{n}}\right)\left(\left(\mathbf{W}^{n}\right)^{H} \overline{\mathbf{H}}^{n} \mathbf{B}^{n}-\mathbf{I}_{N_{b}^{n}}\right)^{H}\right. \\
& \left.\quad+\left(\mathbf{W}^{n}\right)^{H} \mathbf{C}^{n} \mathbf{W}^{n}\right)
\end{aligned}
$$

where we assume that $\mathrm{E}\left[\mathbf{s}^{n}(t)\left(\mathbf{s}^{n}(t)\right)^{H}\right]=\mathbf{I}_{N_{b}^{n}}, n=$ $1, \cdots, N_{c}, \operatorname{tr}(\cdot)$ stands for the matrix trace, and

$$
\begin{aligned}
\overline{\mathbf{H}}^{n} & \triangleq\left[\begin{array}{c}
\mathbf{H}_{r}^{n} \mathbf{F}^{n} \mathbf{H}_{s}^{n} \\
\mathbf{H}_{d}^{n}
\end{array}\right] \\
\mathbf{C}^{n} & \triangleq\left[\begin{array}{cc}
\mathbf{H}_{r}^{n} \mathbf{F}^{n}\left(\mathbf{F}^{n}\right)^{H}\left(\mathbf{H}_{r}^{n}\right)^{H}+\mathbf{I}_{N_{d}} & \mathbf{0} \\
\mathbf{0} & \mathbf{I}_{N_{d}}
\end{array}\right] .
\end{aligned}
$$

The optimal linear receiver that minimizes (8) is the wellknown MMSE receiver, whose weight matrix is given by

$$
\mathbf{W}^{n}=\left[\overline{\mathbf{H}}^{n} \mathbf{B}^{n}\left(\mathbf{B}^{n}\right)^{H}\left(\overline{\mathbf{H}}^{n}\right)^{H}+\mathbf{C}^{n}\right]^{-1} \overline{\mathbf{H}}^{n} \mathbf{B}^{n}, \quad \forall n .
$$

Substituting (9) back into (8), we obtain the minimal MSE as a function of $\mathbf{B}^{n}$ and $\mathbf{F}^{n}, i=1, \cdots, N_{c}$ given by

$$
\begin{aligned}
& \mathrm{MSE}= \\
& \sum_{n=1}^{N_{c}} \operatorname{tr}\left(\left[\mathbf{I}_{N_{b}^{n}}+\left(\mathbf{B}^{n}\right)^{H}\left(\left(\mathbf{H}_{d}^{n}\right)^{H} \mathbf{H}_{d}^{n}+\left(\mathbf{H}_{s}^{n}\right)^{H}\left(\mathbf{F}^{n}\right)^{H}\left(\mathbf{H}_{r}^{n}\right)^{H}\right.\right.\right. \\
& \left.\left.\left.\quad \times\left(\mathbf{H}_{r}^{n} \mathbf{F}^{n}\left(\mathbf{F}^{n}\right)^{H}\left(\mathbf{H}_{r}^{n}\right)^{H}+\mathbf{I}_{N_{d}}\right)^{-1} \mathbf{H}_{r}^{n} \mathbf{F}^{n} \mathbf{H}_{s}^{n}\right) \mathbf{B}^{n}\right]^{-1}\right) .(10)
\end{aligned}
$$

The optimal design problem can be written as

$$
\begin{aligned}
\min _{\left\{\mathbf{B}^{n}\right\},\left\{\mathbf{F}^{n}\right\}} & \mathrm{MSE} \\
\text { s.t. } & \sum_{n=1}^{N_{c}} \operatorname{tr}\left(\mathbf{B}^{n}\left(\mathbf{B}^{n}\right)^{H}\right) \leq p_{s} \\
& \sum_{n=1}^{N_{c}} \operatorname{tr}\left(\mathbf{F}^{n}\left[\mathbf{H}_{s}^{n} \mathbf{B}^{n}\left(\mathbf{B}^{n}\right)^{H}\left(\mathbf{H}_{s}^{n}\right)^{H}+\mathbf{I}_{N_{r}}\right]\left(\mathbf{F}^{n}\right)^{H}\right) \leq p_{r}
\end{aligned}
$$

where for a matrix $\mathbf{A},\left\{\mathbf{A}^{n}\right\} \triangleq \mathbf{A}^{1}, \mathbf{A}^{2}, \cdots, \mathbf{A}^{N_{c}}$. Here (12) and (13) are constraints for the transmission power at the source and relay nodes, respectively, and $p_{s}>0$ and $p_{r}>0$ are the corresponding available power.

When the direct source-destination channel $\mathbf{H}_{d}^{n}$ is weak enough that can be ignored, it can be shown [7] that the optimal source and relay precoding matrices jointly convert the multicarrier MIMO relay channel into parallel single-input single-output (SISO) relay channels. However, when the direct link can not be ignored, due to the inclusion of the terms $\left(\mathbf{H}_{d}^{n}\right)^{H} \mathbf{H}_{d}^{n}, n=1, \cdots, N_{c}$ into the objective function (11), the optimal $\mathbf{F}^{n}$ and $\mathbf{B}^{n}$ do not have the aforementioned structure as in the case of absent direct link. 


\section{A. Alternating Algorithm}

In this subsection, we develop an alternating algorithm to solve problem (11)-(13). This algorithm conditionally updates $\mathbf{F}^{n}$ and $\mathbf{B}^{n}$ in an iterative fashion. Using the matrix inversion lemma, we can rewrite (10) as

$$
\begin{aligned}
\mathrm{MSE}= & \sum_{n=1}^{N_{c}}\left[\operatorname{tr}\left(\left(\mathbf{E}^{n}\right)^{-1}\right)-\operatorname{tr}\left(\mathbf{H}_{r}^{n} \mathbf{F}^{n} \mathbf{T}_{1}^{n}\left(\mathbf{F}^{n}\right)^{H}\left(\mathbf{H}_{r}^{n}\right)^{H}\right.\right. \\
& \left.\left.\times\left[\mathbf{H}_{r}^{n} \mathbf{F}^{n} \mathbf{T}_{2}^{n}\left(\mathbf{F}^{n}\right)^{H}\left(\mathbf{H}_{r}^{n}\right)^{H}+\mathbf{I}_{N_{d}}\right]^{-1}\right)\right]
\end{aligned}
$$

where

$$
\begin{aligned}
& \mathbf{E}^{n} \triangleq \mathbf{I}_{N_{b}^{n}}+\left(\mathbf{B}^{n}\right)^{H}\left(\mathbf{H}_{d}^{n}\right)^{H} \mathbf{H}_{d}^{n} \mathbf{B}^{n} \\
& \mathbf{T}_{1}^{n} \triangleq \mathbf{H}_{s}^{n} \mathbf{B}^{n}\left(\mathbf{E}^{n}\right)^{-2}\left(\mathbf{B}^{n}\right)^{H}\left(\mathbf{H}_{s}^{n}\right)^{H} \\
& \mathbf{T}_{2}^{n} \triangleq \mathbf{H}_{s}^{n} \mathbf{B}^{n}\left(\mathbf{E}^{n}\right)^{-1}\left(\mathbf{B}^{n}\right)^{H}\left(\mathbf{H}_{s}^{n}\right)^{H}+\mathbf{I}_{N_{r}} .
\end{aligned}
$$

In this alternating algorithm, we first update $\left\{\mathbf{F}^{n}\right\}$ with any given feasible $\left\{\mathbf{B}^{n}\right\}$. This problem can be written as

$$
\begin{aligned}
\max _{\left\{\mathbf{F}^{n}\right\}} \sum_{n=1}^{N_{c}} \operatorname{tr} & \left(\mathbf{M}^{n}\left(\mathbf{T}_{2}^{n}\right)^{-\frac{1}{2}} \mathbf{T}_{1}^{n}\left(\mathbf{T}_{2}^{n}\right)^{-\frac{1}{2}}\left(\mathbf{M}^{n}\right)^{H}\right. \\
& \left.\times\left[\mathbf{M}^{n}\left(\mathbf{M}^{n}\right)^{H}+\mathbf{I}_{N_{d}}\right]^{-1}\right) \\
\text { s.t. } & \sum_{n=1}^{N_{c}} \operatorname{tr}\left(\mathbf{F}^{n}\left[\mathbf{H}_{s}^{n} \mathbf{B}^{n}\left(\mathbf{B}^{n}\right)^{H}\left(\mathbf{H}_{s}^{n}\right)^{H}+\mathbf{I}_{N_{r}}\right]\left(\mathbf{F}^{n}\right)^{H}\right) \leq p_{r}
\end{aligned}
$$

where

$$
\mathbf{M}^{n} \triangleq \mathbf{H}_{r}^{n} \mathbf{F}^{n}\left(\mathbf{T}_{2}^{n}\right)^{\frac{1}{2}}
$$

Let us denote

$$
\begin{aligned}
\left(\mathbf{T}_{2}^{n}\right)^{-\frac{1}{2}} \mathbf{T}_{1}^{n}\left(\mathbf{T}_{2}^{n}\right)^{-\frac{1}{2}} & \triangleq \mathbf{U}_{t}^{n} \boldsymbol{\Lambda}_{t}^{n}\left(\mathbf{U}_{t}^{n}\right)^{H} \\
\mathbf{M}^{n} & \triangleq \mathbf{U}_{m}^{n} \boldsymbol{\Lambda}_{m}^{n}\left(\mathbf{V}_{m}^{n}\right)^{H}
\end{aligned}
$$

as the eigendecomposition of $\left(\mathbf{T}_{2}^{n}\right)^{-\frac{1}{2}} \mathbf{T}_{1}^{n}\left(\mathbf{T}_{2}^{n}\right)^{-\frac{1}{2}}$ and the singularvalue decomposition of $\mathbf{M}^{n}$, respectively. It can be shown from [8] that for the optimal $\mathbf{M}^{n}$ which maximizes (14), there is $\mathbf{V}_{m}^{n}=\mathbf{U}_{t}^{n}$, i.e.,

$$
\mathbf{M}_{0}^{n}=\mathbf{U}_{m}^{n} \Lambda_{m}^{n}\left(\mathbf{U}_{t}^{n}\right)^{H}
$$

The maximum of (14) is

$$
\sum_{n=1}^{N_{c}} \operatorname{tr}\left(\boldsymbol{\Lambda}_{m}^{n} \boldsymbol{\Lambda}_{t}^{n}\left(\boldsymbol{\Lambda}_{m}^{n}\right)^{T}\left[\boldsymbol{\Lambda}_{m}^{n}\left(\boldsymbol{\Lambda}_{m}^{n}\right)^{T}+\mathbf{I}_{N_{d}}\right]^{-1}\right)
$$

where the diagonal elements of $\boldsymbol{\Lambda}_{m}^{n}$ and $\boldsymbol{\Lambda}_{t}^{n}$ are arranged in the same order.

We denote $\mathbf{H}_{r}^{n} \triangleq \mathbf{U}_{r}^{n} \boldsymbol{\Lambda}_{r}^{n}\left(\mathbf{V}_{r}^{n}\right)^{H}$ as the singularvalue decomposition of $\mathbf{H}_{r}^{n}$. By substituting (17) back into (16), we obtain

$$
\mathbf{F}^{n}=\mathbf{V}_{r}^{n}\left(\boldsymbol{\Lambda}_{r}^{n}\right)^{+} \mathbf{P}^{n} \boldsymbol{\Lambda}_{m}^{n}\left(\mathbf{U}_{t}^{n}\right)^{H}\left(\mathbf{T}_{2}^{n}\right)^{-\frac{1}{2}}
$$

where $\mathbf{P}^{n} \triangleq\left(\mathbf{U}_{r}^{n}\right)^{H} \mathbf{U}_{m}^{n}$ is an $N_{d} \times N_{d}$ unitary matrix, and the diagonal elements of $\Lambda_{m}^{n}$ and $\Lambda_{r}^{n}$ are arranged in the same order. Substituting (18) back into the left hand side of the power constraint (15), we have

$$
\sum_{n=1}^{N_{c}} \operatorname{tr}\left(\left(\boldsymbol{\Lambda}_{r}^{n}\right)^{+} \mathbf{P}^{n} \boldsymbol{\Lambda}_{m}^{n} \boldsymbol{\Phi}^{n}\left(\boldsymbol{\Lambda}_{m}^{n}\right)^{T}\left(\mathbf{P}^{n}\right)^{H}\left(\left(\boldsymbol{\Lambda}_{r}^{n}\right)^{+}\right)^{T}\right)
$$

where

$$
\boldsymbol{\Phi}^{n \triangleq}\left(\mathbf{U}_{t}^{n}\right)^{H}\left(\mathbf{T}_{2}^{n}\right)^{-\frac{1}{2}}\left[\mathbf{H}_{s}^{n} \mathbf{B}^{n}\left(\mathbf{B}^{n}\right)^{H}\left(\mathbf{H}_{s}^{n}\right)^{H}+\mathbf{I}_{N_{r}}\right]\left(\mathbf{T}_{2}^{n}\right)^{-\frac{1}{2}} \mathbf{U}_{t}^{n} .
$$

Following the technique used in [7], we should find $\mathbf{P}^{n}$ which minimizes (19). It has been shown in [7] that in the absence of the direct source-destination link, $\boldsymbol{\Phi}^{n}$ is a diagonal matrix, and the closed-form solution for $\mathbf{P}^{n}$ can be obtained. However, when $\mathbf{H}_{d}^{n} \neq \mathbf{0}$, in general $\boldsymbol{\Phi}^{n}$ is not a diagonal matrix. Thus, an analytic solution of $\mathbf{P}^{n}$ is intractable. In the following, we take a suboptimal solution of $\mathbf{P}^{n}=\mathbf{D}_{N_{r}}$, or equivalently $\mathbf{U}_{m}^{n}=\mathbf{U}_{r}^{n} \mathbf{D}_{N_{r}}$. Here $\mathbf{D}_{m}$ denotes an arbitrary $m \times m$ diagonal matrix with unit norm diagonal elements, i.e., $\left|\left[\mathbf{D}_{m}\right]_{i, i}\right|=1, i=1, \cdots, m$. Then the rest of the problem is to optimize $\left\{\boldsymbol{\Lambda}_{m}^{n}\right\}$ which can be formulated as

$$
\begin{aligned}
\max _{\left\{\boldsymbol{\Lambda}_{m}^{n}\right\}} & \sum_{n=1}^{N_{c}} \operatorname{tr}\left(\boldsymbol{\Lambda}_{m}^{n} \boldsymbol{\Lambda}_{t}^{n}\left(\boldsymbol{\Lambda}_{m}^{n}\right)^{T}\left[\boldsymbol{\Lambda}_{m}^{n}\left(\boldsymbol{\Lambda}_{m}^{n}\right)^{T}+\mathbf{I}_{N_{d}}\right]^{-1}\right) \\
\text { s.t. } & \sum_{n=1}^{N_{c}} \operatorname{tr}\left(\left(\boldsymbol{\Lambda}_{r}^{n}\right)^{+} \boldsymbol{\Lambda}_{m}^{n} \boldsymbol{\Phi}^{n}\left(\boldsymbol{\Lambda}_{m}^{n}\right)^{T}\left(\left(\boldsymbol{\Lambda}_{r}^{n}\right)^{+}\right)^{T}\right) \leq p_{r}
\end{aligned}
$$

where the diagonal elements of $\Lambda_{m}^{n}, \Lambda_{t}^{n}, \Lambda_{r}^{n}$, and $\Phi^{n}$ are arranged in the same order. Denoting $\lambda_{m, i}^{n}, i=1, \cdots, L, n=$ $1, \cdots, N_{c}$, as the nonzero singularvalue of $\mathbf{M}^{n}$ with $L \triangleq$ $\min \left(n_{r}, n_{d}\right)$. Problem (20), (21) can be rewritten as

$$
\begin{aligned}
\max _{\left\{\lambda_{m, i}^{n}\right\}} & \sum_{n=1}^{N_{c}} \sum_{i=1}^{L} \frac{\left(\lambda_{m, i}^{n}\right)^{2} \lambda_{t, i}^{n}}{\left(\lambda_{m, i}^{n}\right)^{2}+1} \\
\text { s.t. } & \sum_{n=1}^{N_{c}} \sum_{i=1}^{L} \frac{\left(\lambda_{m, i}^{n}\right)^{2} \phi_{i}^{n}}{\left(\lambda_{r, i}^{n}\right)^{2}} \leq p_{r}, \quad \lambda_{m, i}^{n} \geq 0, \quad \forall i, n
\end{aligned}
$$

where $\left\{\lambda_{m, i}^{n}\right\} \triangleq \lambda_{m, 1}^{1}, \cdots, \lambda_{m, L}^{N_{c}}, \lambda_{r, i}^{n}$ and $\lambda_{t, i}^{n}, i=1, \cdots, L$, are the nonzero singularvalue of $\boldsymbol{\Lambda}_{r}^{n}$ and the nonzero eigenvalue of $\left(\mathbf{T}_{2}^{n}\right)^{-\frac{1}{2}} \mathbf{T}_{1}^{n}\left(\mathbf{T}_{2}^{n}\right)^{-\frac{1}{2}}$, respectively, and $\phi_{i}^{n}$ is the $i$ th diagonal element of $\boldsymbol{\Phi}^{n}$. The solution to problem (22)-(23) is

$$
\lambda_{m, i}^{n}=\sqrt{\left[\lambda_{r, i}^{n} \sqrt{\frac{\lambda_{t, i}^{n}}{\kappa \phi_{i}^{n}}}-1\right]^{\dagger}}, \quad \forall i, n
$$

where for a real-valued number $x,[x]^{\dagger} \triangleq \max (x, 0)$. Here $\kappa>0$ is the solution of the following nonlinear equation

$$
\sum_{n=1}^{N_{c}} \sum_{i=1}^{L} \frac{\phi_{i}^{n}}{\left(\lambda_{r, i}^{n}\right)^{2}}\left[\lambda_{r, i}^{n} \sqrt{\frac{\lambda_{t, i}^{n}}{\kappa \phi_{i}^{n}}}-1\right]^{\dagger}=p_{r} .
$$

The left hand side of (24) is monotonically decreasing with respect to $\kappa$. Thus $\kappa$ can be found, for example, by the bisection method [9].

With known $\left\{\mathbf{F}^{n}\right\}$, the problem of updating $\left\{\mathbf{B}^{n}\right\}$ is formulated as

$$
\begin{aligned}
\min _{\left\{\mathbf{B}^{n}\right\}} & \sum_{n=1}^{N_{c}} \operatorname{tr}\left(\left[\mathbf{I}_{N_{b}^{n}}+\left(\mathbf{B}^{n}\right)^{H} \mathbf{\Psi}_{1}^{n} \mathbf{B}^{n}\right]^{-1}\right) \\
\text { s.t. } & \sum_{n=1}^{N_{c}} \operatorname{tr}\left(\left(\mathbf{B}^{n}\right)^{H} \mathbf{B}^{n}\right) \leq p_{s} \\
& \sum_{n=1}^{N_{c}} \operatorname{tr}\left(\left(\mathbf{B}^{n}\right)^{H} \mathbf{\Psi}_{2}^{n} \mathbf{B}^{n}\right) \leq \bar{p}_{r}
\end{aligned}
$$


where

$$
\begin{aligned}
\mathbf{\Psi}_{1}^{n} \triangleq & \left(\mathbf{H}_{d}^{n}\right)^{H} \mathbf{H}_{d}^{n}+\left(\mathbf{H}_{s}^{n}\right)^{H}\left(\mathbf{F}^{n}\right)^{H}\left(\mathbf{H}_{r}^{n}\right)^{H} \\
& \times\left[\mathbf{H}_{r}^{n} \mathbf{F}^{n}\left(\mathbf{F}^{n}\right)^{H}\left(\mathbf{H}_{r}^{n}\right)^{H}+\mathbf{I}_{N_{d}}\right]^{-1} \mathbf{H}_{r}^{n} \mathbf{F}^{n} \mathbf{H}_{s}^{n} \\
\mathbf{\Psi}_{2}^{n} \triangleq & \left(\mathbf{H}_{s}^{n}\right)^{H}\left(\mathbf{F}^{n}\right)^{H} \mathbf{F}^{n} \mathbf{H}_{s}^{n} \\
\bar{p}_{r} \triangleq & p_{r}-\sum_{n=1}^{N_{c}} \operatorname{tr}\left(\mathbf{F}^{n}\left(\mathbf{F}^{n}\right)^{H}\right) .
\end{aligned}
$$

Problem (25)-(27) can be solved by the Lagrange multiplier method [9]. The Lagrangian function is given by

$\mathcal{L}=\sum_{n=1}^{N_{c}} \operatorname{tr}\left(\left[\mathbf{I}_{N_{b}^{n}}+\left(\mathbf{B}^{n}\right)^{H} \mathbf{\Psi}_{1}^{n} \mathbf{B}^{n}\right]^{-1}\right)+$

$\eta_{1}\left(\sum_{n=1}^{N_{c}} \operatorname{tr}\left(\left(\mathbf{B}^{n}\right)^{H} \mathbf{B}^{n}\right)-p_{s}\right)+\eta_{2}\left(\sum_{n=1}^{N_{c}} \operatorname{tr}\left(\left(\mathbf{B}^{n}\right)^{H} \mathbf{\Psi}_{2}^{n} \mathbf{B}^{n}\right)-\bar{p}_{r}\right)$

where $\eta_{1} \geq 0$ and $\eta_{2} \geq 0$ are the Lagrangian multipliers associated with the constraints (26) and (27), respectively. Taking the derivative of $\mathcal{L}$ with respect to $\mathbf{B}^{n}$ and letting the result be 0 , it can be shown that the optimal $\mathbf{B}^{n}$ satisfies the following matrix equation

$$
\begin{aligned}
& \left(\mathbf{B}^{n}\right)^{H} \mathbf{\Psi}_{1}^{n} \mathbf{B}^{n}\left[\mathbf{I}_{N_{b}^{n}}+\left(\mathbf{B}^{n}\right)^{H} \mathbf{\Psi}_{1}^{n} \mathbf{B}^{n}\right]^{-2} \\
& =\left(\mathbf{B}^{n}\right)^{H}\left(\eta_{1} \mathbf{I}_{N_{s}}+\eta_{2} \mathbf{\Psi}_{2}^{n}\right) \mathbf{B}^{n}, \quad n=1, \cdots, N_{c} .
\end{aligned}
$$

Let us denote

$$
\begin{aligned}
& \overline{\mathbf{B}}^{n} \triangleq\left(\mathbf{\Psi}_{1}^{n}\right)^{\frac{1}{2}} \mathbf{B}^{n} \\
& \boldsymbol{\Theta}^{n} \triangleq\left(\Psi_{1}^{n}\right)^{-\frac{1}{2}}\left(\eta_{1} \mathbf{I}_{N_{s}}+\eta_{2} \Psi_{2}^{n}\right)\left(\Psi_{1}^{n}\right)^{-\frac{1}{2}} .
\end{aligned}
$$

Then (28) can be rewritten as

$$
\left(\overline{\mathbf{B}}^{n}\right)^{H} \overline{\mathbf{B}}^{n}=\left(\overline{\mathbf{B}}^{n}\right)^{H} \boldsymbol{\Theta}^{n} \overline{\mathbf{B}}^{n}\left[\mathbf{I}_{N_{b}^{n}}+\left(\overline{\mathbf{B}}^{n}\right)^{H} \overline{\mathbf{B}}^{n}\right]^{2} .
$$

Denoting $\boldsymbol{\Theta}^{n} \triangleq \mathbf{U}_{\theta}^{n} \boldsymbol{\Lambda}_{\theta}^{n}\left(\mathbf{U}_{\theta}^{n}\right)^{H}$ as the eigendecomposition of $\Theta^{n}$, we obtain the solution to (29) as $\overline{\mathbf{B}}^{n}=\mathbf{U}_{\theta}^{n} \overline{\mathbf{\Lambda}}_{b}^{n}\left(\overline{\mathbf{V}}_{b}^{n}\right)^{H}$, or equivalently

$$
\mathbf{B}^{n}=\left(\mathbf{\Psi}_{1}^{n}\right)^{-\frac{1}{2}} \mathbf{U}_{\theta}^{n} \overline{\mathbf{\Lambda}}_{b}^{n}\left(\overline{\mathbf{V}}_{b}^{n}\right)^{H}
$$

where $\overline{\mathbf{V}}_{b}^{n}$ is an arbitrary $N_{b}^{n} \times N_{b}^{n}$ unitary matrix, and $\overline{\boldsymbol{\Lambda}}_{b}^{n}$ is the singularvalue matrix of $\overline{\mathbf{B}}^{n}$ satisfying

$$
\left(\overline{\boldsymbol{\Lambda}}_{b}^{n}\right)^{T} \overline{\boldsymbol{\Lambda}}_{b}^{n}=\left(\overline{\boldsymbol{\Lambda}}_{b}^{n}\right)^{T} \boldsymbol{\Lambda}_{\theta}^{n} \overline{\boldsymbol{\Lambda}}_{b}^{n}\left[\mathbf{I}_{N_{b}^{n}}+\left(\overline{\boldsymbol{\Lambda}}_{b}^{n}\right)^{T} \overline{\boldsymbol{\Lambda}}_{b}^{n}\right]^{2} .
$$

Solving (31), we obtain

$$
\bar{\lambda}_{b, i}^{n}=\sqrt{\left[\sqrt{1 / \lambda_{\theta, i}^{n}}-1\right]^{\dagger},} \quad i=1, \cdots, N_{b}^{n}
$$

where $\bar{\lambda}_{b, i}^{n}$ is the $i$ th singularvalue of $\overline{\mathbf{B}}^{n}$, and $\lambda_{\theta, i}^{n}$ denotes the $i$ th eigenvalue of $\boldsymbol{\Theta}^{n}$. Note that $N_{b}^{n}, n=1, \cdots, N_{c}$ are automatically determined by our algorithm. The Lagrangian multipliers $\eta_{1}$ and $\eta_{2}$ can be obtained by substituting (30) back into (26) and (27) and solving the following system of nonlinear equations

$$
\begin{aligned}
\sum_{n=1}^{N_{c}} \operatorname{tr}\left(\left(\boldsymbol{\Psi}_{1}^{n}\right)^{-1}\left(\left[\eta_{1}\left(\mathbf{\Psi}_{1}^{n}\right)^{-1}+\eta_{2} \boldsymbol{\Gamma}^{n}\right]^{-\frac{1}{2}}-\mathbf{I}_{N_{s}}\right)^{\ddagger}\right) & =p_{s} \\
\sum_{n=1}^{N_{c}} \operatorname{tr}\left(\boldsymbol{\Gamma}^{n}\left(\left[\eta_{1}\left(\boldsymbol{\Psi}_{1}^{n}\right)^{-1}+\eta_{2} \boldsymbol{\Gamma}^{n}\right]^{-\frac{1}{2}}-\mathbf{I}_{N_{s}}\right)^{\ddagger}\right) & =\bar{p}_{r}
\end{aligned}
$$

where $\boldsymbol{\Gamma}^{n} \triangleq\left(\boldsymbol{\Psi}_{1}^{n}\right)^{-\frac{1}{2}} \boldsymbol{\Psi}_{2}^{n}\left(\boldsymbol{\Psi}_{1}^{n}\right)^{-\frac{1}{2}}$. Here for a matrix $\mathbf{Z}$, $\mathbf{Z}^{\ddagger}$ denotes projecting $\mathbf{Z}$ to the set of positive semi-definite matrices with commensurate dimension. Equations (32), (33) can be solved by the two-dimensional bisection method [10].

Note that under $\mathbf{P}^{n}=\mathbf{D}_{N_{r}}, n=1, \cdots, N_{c}$, the conditional updates of $\left\{\mathbf{F}^{n}\right\}$ and $\left\{\mathbf{B}^{n}\right\}$ may either decrease or maintain but cannot increase the MSE. Monotonic convergence of the proposed alternating algorithm can be achieved.

\section{B. Suboptimal Non-Alternating Algorithm}

As will be seen in Section IV, the alternating algorithm we developed greatly reduces the MSE of the signal waveform estimation. However, it may be computationally intensive due to the iterative nature of the algorithm. In practice, in order to reduce the computational complexity, one can use a suboptimal non-alternating algorithm. In particular, we use the source precoding matrices $\left\{\mathbf{B}^{n}\right\}$ developed for the case of no direct source-destination link [7], given by

$$
\mathbf{B}^{n}=\mathbf{V}_{s}^{n} \boldsymbol{\Lambda}_{b}^{n} \mathbf{V}_{b}^{n}
$$

where $\mathbf{V}_{s}^{n}$ is the right singularvector matrix of $\mathbf{H}_{s}^{n}, \mathbf{V}_{b}^{n}$ is an arbitrary $N_{b}^{n} \times N_{b}^{n}$ unitary matrix, and $\boldsymbol{\Lambda}_{b}^{n}$ is the singular value matrix of $\mathbf{B}^{n}$. Then we only need to optimize $\left\{\mathbf{F}^{n}\right\}$ by solving problem (14), (15) with matrices $\left\{\mathbf{B}^{n}\right\}$ obtained in (34). It can be seen in Section IV that this suboptimal algorithm has a mild performance degradation but substantially reduced computational complexity compared with the alternating algorithm.

\section{Simulations}

In this section, we study the performance of the proposed non-regenerative multicarrier MIMO relay techniques through numerical simulations. For all examples, the channel between each transmit-receive antenna pair is modelled as the ETSI "Vehicular A" multipath channel environment [11]. An OFDM communication system with $N_{c}=64$ subcarriers is assumed. The channel matrices have zero-mean entries with variances $\sigma_{s}^{2}, \sigma_{r}^{2}, \sigma_{d}^{2}$ for $\mathbf{H}_{s}^{n}, \mathbf{H}_{r}^{n}$, and $\mathbf{H}_{d}^{n}$, respectively. The signal-tonoise ratios (SNRs) are defined as

$$
\mathrm{SNR}_{\mathrm{s}} \triangleq \frac{\sigma_{s}^{2} p_{s}}{N_{c}}, \quad \mathrm{SNR}_{\mathrm{r}} \triangleq \frac{\sigma_{r}^{2} p_{r}}{N_{c}}, \quad \mathrm{SNR}_{\mathrm{d}} \triangleq \frac{\sigma_{d}^{2} p_{s}}{N_{c}}
$$

for the source-relay, relay-destination, and source-destination links, respectively. The averaged MSE (AMSE) is computed as

$$
\operatorname{AMSE}=\frac{\sum_{q=1}^{N_{q}} \sum_{n=1}^{N_{c}} \operatorname{tr}\left(\left[\hat{\mathbf{s}}^{n}(q)-\mathbf{s}^{n}(q)\right]\left[\hat{\mathbf{s}}^{n}(q)-\mathbf{s}^{n}(q)\right]^{H}\right)}{N_{q} N_{c} N_{s}}
$$

where the average is carried out over $N_{q}=2000$ independent channel realizations.

We compare the proposed alternating algorithm and the suboptimal algorithm with the following schemes.

- Relay-only suboptimal (ROS) algorithm: In this scheme, one chooses an identity source precoding matrix

$$
\mathbf{B}^{n}=\sqrt{\frac{p_{s}}{N_{s} N_{c}}} \mathbf{I}_{N_{s}}, \quad \forall n
$$


and $\mathbf{F}^{n}$ is given in [7].

- Naive amplify-and-forward (NAF) algorithm: Here $\mathbf{B}^{n}$ is given by (35), while $\mathbf{F}^{n}$ is taken as

$$
\mathbf{F}^{n}=\sqrt{\frac{p_{r}}{N_{c} \operatorname{tr}\left(\Upsilon^{n}\right)}} \mathbf{I}_{N_{r}}, \quad \forall n
$$

where $\boldsymbol{\Upsilon}^{n} \triangleq \frac{p_{s}}{N_{s} N_{c}} \mathbf{H}_{s}^{n}\left(\mathbf{H}_{s}^{n}\right)^{H}+\mathbf{I}_{N_{r}}, \quad \forall n$.

- Pseudo matched-filter (PMF) algorithm [12]: In this scheme, $\mathbf{B}^{n}$ is given by (35), and $\mathbf{F}^{n}$ is

$$
\mathbf{F}^{n}=\sqrt{\frac{p_{r}}{N_{c} \operatorname{tr}\left(\left(\mathbf{H}_{r}^{n}\right)^{H}\left(\mathbf{H}_{s}^{n}\right)^{H} \mathbf{\Upsilon}^{n} \mathbf{H}_{s}^{n} \mathbf{H}_{r}^{n}\right)}}\left(\mathbf{H}_{s}^{n} \mathbf{H}_{r}^{n}\right)^{H}, \quad \forall n .
$$

- Optimal algorithm designed for the absence of the direct source-destination link (OAD) given in [7]. Here the source and relay precoding matrices jointly diagonalize the source-relay-destination channel.

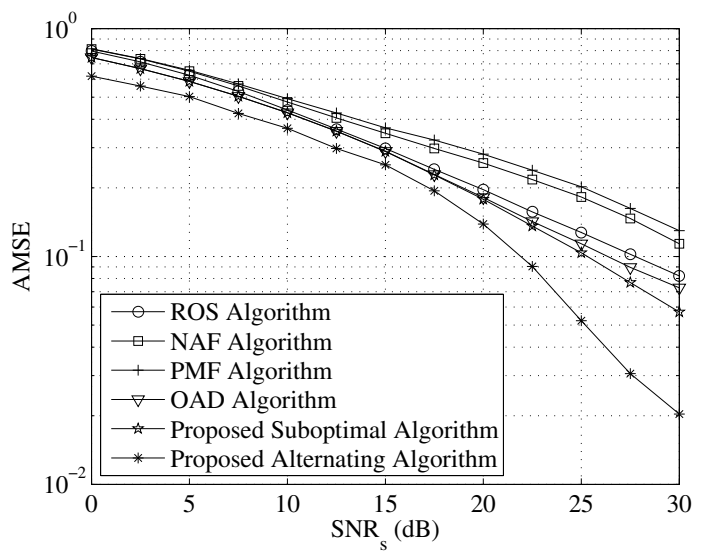

Fig. 1. AMSE versus $\mathrm{SNR}_{\mathrm{s}} . N_{s}=N_{r}=N_{d}=3, \mathrm{SNR}_{\mathrm{r}}=20 \mathrm{~dB}$, $\mathrm{SNR}_{\mathrm{d}}=0 \mathrm{~dB}$.

In our first example, we choose $N_{s}=N_{r}=N_{d}=3$, $\mathrm{SNR}_{\mathrm{r}}=20 \mathrm{~dB}$, and $\mathrm{SNR}_{\mathrm{d}}=0 \mathrm{~dB}$. Fig. 1 displays the AMSE of all algorithms versus $\mathrm{SNR}_{\mathrm{s}}$. It can be seen that the proposed alternating algorithm consistently yields the smallest AMSE over the whole $\mathrm{SNR}_{\mathrm{s}}$ range. The proposed suboptimal algorithm trades a reduced performance for a computational complexity reduction. Although the OAD algorithm is MSEoptimal in the absence of the direct source-destination link, it yields a larger AMSE in the presence of the direct link compared with the proposed algorithms.

In the second example, we set $N_{s}=5, N_{r}=6, N_{d}=4$, $\mathrm{SNR}_{\mathrm{r}}=20 \mathrm{~dB}$, and fix $\mathrm{SNR}_{\mathrm{d}}$ to $10 \mathrm{~dB}$. The AMSEs of all algorithms except the PMF scheme versus $\mathrm{SNR}_{\mathrm{S}}$ are displayed in Fig. 2. Note that in contrast to other schemes, the PMF algorithm requires $N_{d}=N_{s}$. We find that the proposed alternating scheme has the best performance. The performance of the ROS and NAF algorithms degrade significantly when all nodes have different number of antennas, because in these two schemes, the source precoding matrix is "omnidirectional".

From Figs. 1 and 2 we find that an efficient exploitation of the direct source-destination link is very important. The direct link increases the spatial diversity of the whole system, and therefore, greatly reduces the AMSE.

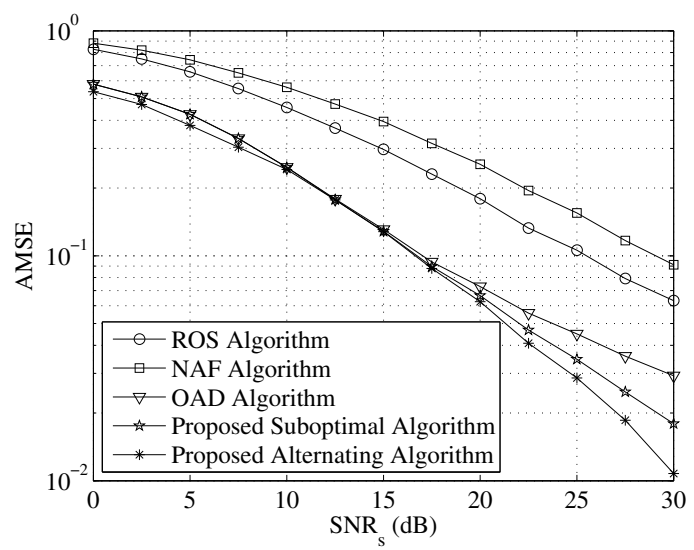

Fig. 2. AMSE versus $\mathrm{SNR}_{\mathrm{s}} . N_{s}=5, N_{r}=6, N_{d}=4, \mathrm{SNR}_{\mathrm{r}}=20 \mathrm{~dB}$, $\mathrm{SNR}_{\mathrm{d}}=10 \mathrm{~dB}$

\section{CONCLUSiOnS}

We developed novel non-regenerative (linear precoding) techniques for multicarrier MIMO wireless relay communications in the presence of the direct source-destination link. The proposed source and relay precoding matrices efficiently exploit the direct link, and jointly minimize the MSE of the signal waveform estimation. We observed that the direct link provides valuable spatial diversity to the entire relay communication system.

\section{REFERENCES}

[1] T. M. Cover and A. A. El Gamal, "Capacity theorems for the relay channel," IEEE Trans. Inform. Theory, vol. 25, pp. 572-584, Sep. 1979.

[2] A. Sendonaris, E. Erkip, and B. Aazhang, "User cooperation diversity - part I and part II," IEEE Trans. Commun., vol. 51, pp. 1927-1948, Nov. 2003.

[3] J. N. Laneman, D. N. C. Tse, and G. W. Wornell, "Cooperative diversity in wireless networks: Efficient protocols and outage behavior," IEEE Trans. Inform. Theroy, vol. 50, pp. 3062-3080, Dec. 2004.

[4] X. Tang and Y. Hua, "Optimal design of non-regenerative MIMO wireless relays," IEEE Trans. Wireless Commun., vol. 6, pp. 1398-1407, Apr. 2007.

[5] O. Muñoz-Medina, J. Vidal, and A. Agustín, "Linear transceiver design in nonregenerative relays with channel state information," IEEE Trans. Signal Processing, vol. 55, pp. 2593-2604, Jun. 2007.

[6] I. Hammerström and A. Wittneben, "Joint power allocation for nonregenerative MIMO-OFDM relay links," in Proc. IEEE Intern. Conf. Acoustics, Speech, and Signal Processing, Toulouse, France, May 2006, vol. 4, pp. 49-52.

[7] Y. Rong, "Non-regenerative multicarrier multiple-input multiple-output relay communications based on minimization of mean-squared error," submitted to IEEE Trans. Signal Processing.

[8] A. W. Marshall and I. Olkin, Inequalities: Theory of Majorization and Its Applications, Academic Press, 1979.

[9] S. Boyd and L. Vandenberghe, Convex Optimization. Cambridge, U.K.: Cambridge University Press, 2004.

[10] R. B. Kearfott, "Abstract generalized bisection and a cost bound," Math. Comput., vol. 49, pp. 187-202, Jul. 1987.

[11] "Universal Mobile Telecommunications System (UMTS); Selection Procedures for the Choice of Radio Transmission Technologies of the UMTS (UMTS 30.03 Version 3.2.0)," ETSI Standard, Document TR 101112 V3.2.0, 1998

[12] P. U. Sripathi and J. S. Lehnert, "A throughput scaling law for a class of wireless relay networks," in Proc. 38th Annual Asilomar Conference on Signals, Systems and Computers, Nov. 2004, vol. 2, pp. 1333-1337. 